\title{
Eigenschaften einer infektiösen Nucleinsäure-Fraktion aus Hühnerembryonen, die mit Encephalitis-Virus infiziert wurden
}

\author{
I. Mitt.: Physikalische und chemische Eigenschaften
}

\author{
Von Eberhard Wecker
}

\author{
Aus dem Max-Planck-Institut für Virusforschung, Tübingen, Abteilung für tierpathogene Virusarten * \\ (Z. Naturforschg. 14 b, 370-378 [1959]; eingegangen am 12. Februar 1959)
}

\begin{abstract}
Aus Homogenaten von Hühnerembryonen, die an einer Infektion mit EEE-Virus ** zugrunde gegangen sind, lassen sich durch Behandlung mit Phenol bei niederen Temperaturen Nucleinsäure-Fraktionen gewinnen (NS-Fraktionen), die infektiös sind. Ihr Nucleinsäureanteil setzt sich zu etwa $75 \%$ aus Ribonucleinsäure und zu etwa 25\% aus Desoxyribonucleinsäure zusammen. Außer dem Zuckeranteil der beiden Nucleinsäure-Typen besitzen sie noch andere, hochmolekulare Zuckerkomponenten. Weitere Verunreinigungen der Präparate durch Peptide oder Aminosäuren lassen sich durch AlkoholPräzipitation der Nucleinsäuren entfernen. Die Infektiosität der NS-Fraktionen geht auf ihren Ribonucleinsäure (RNS) -Anteil zurück. Dieser ist aus Molekülen unterschiedlicher Größe zusammengesetzt, die infektiöse RNS ist jedoch nach ihrer Sedimentationskonstante hochmolekular. Ihr Mol.-Gew. beträgt wahrscheinlich $2 \cdot 10^{6}$.
\end{abstract}

Die von Gierer und Schramm angegebene Methode, mit der sie aus Tabakmosaik-Virus (TMV) mittels Phenol eine infektiöse RNS isolieren konnten ${ }^{1}$, wurde auch schon bei tier- und menschenpathogenen Virusarten von Colter und Mitarb. ${ }^{2,3}$ und WECKER und $\mathrm{S}_{\mathrm{CHÄFER}}{ }^{4}$ erfolgreich angewendet. Allerdings wurden hier die NS-Fraktionen nicht aus dem Virus selbst, sondern aus virus-infizierten Zellen oder Geweben gewonnen. Neuerdings gelang auch die Herstellung von infektiösen RNS-Präparaten aus angereichertem Polio-Virus mit dieser Technik ${ }^{5}$.

Entsprechend dem komplexeren Ausgangsmaterial sind diese infektiösen NS-Fraktionen auch weniger einheitlich als z. B. bei TMV und der Beweis, daß es sich bei dem infektiösen Agens um freie RNS-Moleküle handelt, wurde bisher noch nicht schlüssig erbracht. Auch fehlen noch detailliertere physikalische und chemische Untersuchungen.

Die Absicht der vorliegenden Arbeit ist es, Beweise dafür zu erbringen, daß es sich bei dem infektiösen Material in NS-Fraktionen aus virus-infizierten Hühnerembryonen um freie RNS-Moleküle handelt. Die physikalischen und chemischen Eigenschaften der NS-Fraktionen werden eingehender studiert.

* Gegenwärtige Anschrift: The Wistar Institute, Philadelphia, Pennsylvania/USA.

* EEE-Virus = eastern equine encephalomyelitis virus = Virus der amerikanischen Pferde-Encephalomyelitis (Typ Ost).

1 A. Gierer u. G. Schramm, Nature [London] 177, 702 [1956] ; Z. Naturforschg. 11 b, 138 [1956].

\section{Material und Methoden}

1. Gewinnung der virus-infizierten
Embryonen

10 bis 11 Tage lang bebrütete, befruchtete Hühnereier wurden mit je $0,2 \mathrm{~cm}^{3}$ EEE-Virus-haltiger Lösung infiziert, die ca. $20 \mathrm{LD}_{50}$ enthielten. Die Lösung wurde in die Allantoishöhle injiziert. Die innerhalb 24 bis $48 \mathrm{Stdn}$. abgestorbenen Embryonen wurden möglichst unmittelbar nach dem Tode entnommen und dann sofort bei $-40{ }^{\circ} \mathrm{C}$ eingefroren.

\section{Gew innung der NS-Fraktionen}

4 gefrorene Embryonen wurden mit $12 \mathrm{~cm}^{3}$ 0,02-m. Phosphatpuffer $p_{\mathrm{H}} 7,0$ und $20 \mathrm{~cm}^{3}$ 80-proz. Phenol in einem eisgekühlten Homogenisator zerkleinert. Die Homogenate wurden anschließend $3 \mathrm{Min}$. bei 13000 Umdrehungen pro Min. (U/Min.) und etwa $+14{ }^{\circ} \mathrm{C}$ zentrifugiert und die obere Wasserphase abgezogen. Diese wurde mit $20 \mathrm{~cm}^{3}$ Phenol in der Kälte für 8 Min. möglichst hart und frequent in einer Maschine geschüttelt (ca. 250 bis 300 Hübe/Min.) und anschließend wieder 3 Min. wie oben zentrifugiert. Das Schütteln der Wasserphase mit Phenol wurde insgesamt 3-mal durchgeführt.

Die zuletzt gewonnene Wasserphase wurde 6-mal mit einem jeweils überschüssigen Volumen von peroxydfreiem Äther mit der Hand durchgeschüttelt, um das

2 J. S. Colter, H. H. Birs u. R. A. Brown, Nature [London] 179, 859 [1957].

3 J. S. Colter, H. H. Bird, A. W. Moyer u. R. A. Brown, Virology 4, 522 [1957].

${ }^{4}$ E. WECKER u. W. SCHÄFER, Z. Naturforschg. 12 b, 415 [1957].

5 H. E. Alexander, G. Koch, J. M. Mountain, K. Sprunt u. O. van Damme, Virology 5, 172 [1958]. 
restliche Phenol zu entfernen. Zum Schluß wurde der restliche Äther im Stickstoffstrom ausgetrieben. Sämtliche Manipulationen wurden möglichst bei $+4{ }^{\circ} \mathrm{C}$ oder unter Kühlung im Eisbad durchgeführt. Die auf die beschriebene Weise gewonnenen Produkte sind im folgenden als ,rohe NS-Fraktionen“ bezeichnet.

\section{Alkohol-Präzipitation der NS-Fraktionen}

1 Volumenteil rohe NS-Fraktion wurde mit 2 Volumenteilen 96-proz. Äthanol im Eisbad versetzt. Die sofort auftretenden Ausfällungen wurden in einer gekühlten Zentrifuge bei 3000 U/Min. kurz abgeschleudert und die Überstände dekantiert. Das Sediment wurde in einer eisgekühlten Mischung aus 1 Volumen 0,02-m. Phosphatpuffer $p_{\mathrm{H}} 7,0$ und 2 Volumen Äthanol mit einer Pipette kräftig suspendiert und wieder abgeschleudert. Nach nochmaligem Waschen wurde dann das Sediment im ursprünglichen oder einem geringeren Volumen 0,02-m. Phosphatpuffer gelöst. Diese Produkte werden im Text als „alkoholpräzipitierte NS-Fraktionen“ bezeichnet.

\section{Messung der U V-Spektren}

Die Messung der UV-Spektren wurde mit NS-Fraktionen durchgeführt, die $1: 50$ oder $1: 100$ in 0,1-n. Phosphatpuffer $p_{\mathrm{H}} 7,5$ verdünnt worden waren. Die Messungen erfolgten in einem UV-Spektrophotometer der Firma Zeiss.

5. Bestimmung der

Sedimentationskonstanten

Die Bestimmung der Sedimentationskonstanten wurde in einer analytischen Ultrazentrifuge der Firma Phywe, Göttingen, bei etwa $+4{ }^{\circ} \mathrm{C}$ durchgeführt. Die NS-Fraktionen waren dabei in 0,02-m. Phosphatpuffer $p_{\mathrm{H}} 7,0$ gelöst.

\section{Bestimmung der Viskosität}

Die Viskositäten wurden in einem $\mathrm{Ostw}$ a ld-Viskosimeter bei $+4{ }^{\circ} \mathrm{C}$ gemessen. Die angegebenen Durchlaufzeiten sind Mittelwerte von Doppelbestimmungen. Die Ablesedifferenz beträgt dabei maximal $\pm 0,5$ Sekunden.

\section{Protein-Nach weis}

Der Protein-Nachweis erfolgte nach der von MaRкнам und Sмiтн $^{6}$ beschriebenen modifizierten Biuret-Reaktion.

$$
\begin{aligned}
& \text { 8. Desoxyribonucleinsäure (DNS) - } \\
& \text { B estimmung }
\end{aligned}
$$

Die DNS-Bestimmungen wurden in der von Hershey et al. ${ }^{7}$ beschriebenen Weise mit D i s c h e s Reagens auf Desoxyribose durchgeführt. $1 \mathrm{~cm}^{3}$ NS-Fraktion wurde mit $2 \mathrm{~cm}^{3}$ D i s ches Reagens für $20 \mathrm{Min}$. im kochenden Wasserbad erhitzt, anschließend noch 30 Min. bei Raumtemperatur gehalten. Die Extinktion der auftretenden Blaufärbung wurde in einem Photometer „Eppendorf“ bei $578 \mathrm{~m} \mu$ Wellenlänge gemessen. Der DNS.
Gehalt wurde dann von einer Eichkurve abgelesen, die unter Verwendung von eingewogenen Mengen DNS aufgenommen worden war.

\section{Zucker-Reaktion}

Die Gesamt-Zucker-Bestimmung wurde mit der OrcinReaktion nach Tilmans und PhilipPi ${ }^{8}$ durchgeführt. Die Werte wurden nach Bestimmung der Extinktion ebenfalls von einer Eichkurve abgelesen und sind als Glucose-Äquivalente angegeben.

\section{Stickst of f-Bestim mung}

Die Stickstoff-Bestimmung erfolgte nach der Mikromethode von $\mathrm{K}$ j e l d a h l.

\section{Enz y m e}

Als Enzyme wurden Präparate von kristallisierter Ribonuclease (RNase) bzw. Desoxyribonuclease (DNase) der Firma Worthington verwendet.

\section{Ergebnisse}

I. Physikalische Eigenschaften der NS-Fraktionen

\section{Die UV-Absorption}

Die UV-Absorptions-Kurven der NS-Fraktionen zeigten immer den für Nucleinsäure typischen Verlauf. Das Absorptions-Maximum lag bei $258 \mathrm{~m} \mu$, das Minimum bei $230 \mathrm{~m} \mu$ (s. Abb. 1).

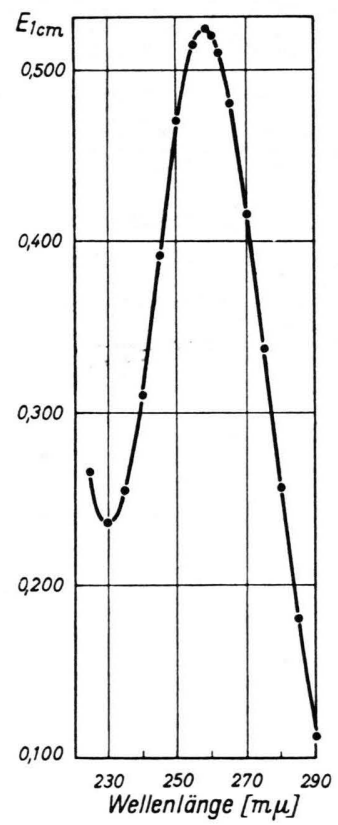

Abb. 1. UV-Absorptions-Spektrum einer alkoholpräzipitierten NS-Fraktion. 
Das Verhältnis der Extinktionen E $258 \mathrm{~m} \mu$ / E $280 \mathrm{~m} \mu$ gibt einen Anhalt für Verunreinigungen der Nucleinsäuren z. B. mit Proteinen. Bei den nicht durch Alkohol-Präzipitation gereinigten rohen NSFraktionen ergab sich der durchschnittliche Wert von $1,95$ (2,01 bis 1,88$)$, bei den alkohol-präzipitierten der Durchschnittswert von 2,06 (2,19 bis 1,95). Das Verhältnis wurde regelmäßig durch die Fällung mit Alkohol zu höheren Werten verschoben. Dies zeigt an, daß Verunreinigungen, die bei $280 \mathrm{~m} \mu$ absorbieren, dadurch entfernt werden konnten. Wenn es sich dabei um Peptide handelte, so müßten diese relativ klein sein, da größere Peptidmoleküle durch Alkohol ebenfalls präzipitiert werden.

Aus der Extinktion einer NS-Fraktion bei der Wellenlänge $258 \mathrm{~m} \mu$ läßt sich die Konzentration an NS errechnen. Bei einer Schichtdicke von $1 \mathrm{~cm}$ wird pro $\gamma$ NS-Stickstoff eine Extinktion von $E_{258 \mathrm{~m} \mu}^{1 \mathrm{~cm}}=0,150$ angenommen, ein Wert, der z. B. bei der RNS aus TMV experimentell ermittelt wurde (vgl. l. c. ${ }^{9}$ ). Dies entspricht einem Extinktions-Koeffizienten $E_{1 \mathrm{~cm}^{3}}^{1 \%}=250$, wenn für die NS ein Stickstoff-Gehalt von $16,7 \%=1 / 6$ berechnet wird, was bei einer RNS mit äquimolarem Basenverhältnis der Fall ist.

Von Colter und Brown ${ }^{10}$ wurde für die RNS aus Ehrlich-Ascites-Tumor-Zellen ein Koeffizient $E_{1 \mathrm{~cm}^{3}}^{1 \%}$ $=210$ angegeben. Dieser Wert ist deshalb so niedrig, weil die Autoren bei ihrer Rechnung nur 14\% Stickstoff-Gehalt der RNS, also weniger als $1 / 7$ annahmen. Rechnet man dagegen auch hier $16,7 \%$ Stickstoff/RNS-Molekül, so ergibt sich genau der Wert $E_{1}^{1 \%} \mathrm{~cm}^{3}=250$, was bedeutet, daß auch Colter und Brown pro $\gamma$ RNS-Stickstoff einen Wert von 0,150 einsetzten.

Vergleicht man die gemäß der Extinktion bei $258 \mathrm{~m} \mu$ berechneten Stickstoff-Werte mit denjenigen, die durch direkte Stickstoff-Bestimmung ermittelt wurden, so muß sich ein Maß für die Verunreinigung des Nucleinsäure-Präparates mit stickstoffhaltigen anderen Substanzen ergeben.

Der Vergleich wurde bei einigen NS-Fraktionen vor und nach der Alkohol-Präzipitation durchgeführt (s. Tab. 1). Die Ergebnisse sprechen eindeutig dafür, daß durch die Fällung mit Alkohol, stickstoffhaltige Begleitsubstanzen, die vorwiegend bei $280 \mathrm{~m} \mu$ ab-

${ }_{6}^{6}$ R. Markham u. J. D. Smith, J. Biochemistry [Tokyo] 46, 509 [1950].

7 A. D. Hershey, J. Dixon u. M. Chase, J. gen. Physiol. 36, 777 [1953].

\begin{tabular}{|l|c|c|c|}
\hline Präparat & $\begin{array}{c}\mathrm{N} \text { berechnet } \\
{\left[\gamma / \mathrm{cm}^{3}\right]}\end{array}$ & $\begin{array}{c}\mathrm{N} \text { bestimmt } \\
{\left[\gamma / \mathrm{cm}^{3}\right]}\end{array}$ & $\begin{array}{c}\text { Verunreini- } \\
\text { gung } \\
{[\%]}\end{array}$ \\
\hline Vor & 310 & 506 & 39 \\
Präzipi- & 323 & 578 & 44 \\
tation & 417 & 815 & 49 \\
Nach & 195 & 196 & 0 \\
Präzipi- & 359 & 358 & 0 \\
tation & 480 & 488 & 1,6 \\
& 405 & 380 & 0 \\
& 407 & 425 & 4 \\
\hline
\end{tabular}

Tab. 1. Berechnete und bestimmte $\mathrm{N}_{2}$-Menge in NS-Fraktionen vor und nach der Alkohol-Präzipitation.

sorbieren, nahezu vollständig aus der NS-Fraktion entfernt werden können.

\section{Die Viskosität}

Für eine Mol.-Gew.-Bestimmung sind bei bekannter Dichte mindestens zwei weitere Parameter erforderlich, z. B. die Viskositätszahl und die Sedimentationskonstante. Unter Verwendung dieser beiden Parameter hat GIERER das Mol.-Gew. der infektiösen RNS aus TMV bestimmt ${ }^{9}$.

Es war von Interesse, entsprechende Untersuchungen auch mit den hier vorliegenden infektiösen NSFraktionen durchzuführen.

Die Viskositätszahl $[\eta]$ wurde auf das Volumen der gelösten Nucleinsäure bezogen; sie ist nach der folgenden Gleichung in dimensionslosen Einheiten definiert:

$$
[\eta]=\frac{\varrho}{c}\left(\frac{\eta}{\eta_{0}}-1\right)
$$

( $\varrho=$ Dichte $; c=$ Konzentration der NS; $\eta=$ Viskosität bei der Konzentration $c$ in $\mathrm{g} / 1 \mathrm{~cm}^{3}, \eta_{\mathbf{0}}=$ Viskosität des reinen Lösungsmittels) .

Eine alkohol-präzipitierte NS-Fraktion mit einer Konzentration von $0,86 \mathrm{mg} / \mathrm{cm}^{3}$ hatte eine für diese NS-Konzentration berechnete Viskositätszahl $[\eta]=1120$, d. h. die etwa 10 -fache Viskosität einer RNS aus TMV gleicher Konzentration ${ }^{9}$. Es war also damit zu rechnen, daß neben der RNS in den NSFraktionen noch andere, höher visköse Substanzen vorliegen.

Der Beitrag des RNS-Anteiles zu der gesamten Viskosität einer NS-Fraktion wurde auf die folgende

8 J. Tillmanss u. K. Philippi, Z. Biochem. 215, 36 [1929].

9 A. Gierer, Z. Naturforschg. 13 b, 477 [1958].

10 J. S. Colter u. R. A. Brown, Science [Washington] 124, 1077 [1956]. 
Weise ermittelt: Nach Bestimmung der Durchlaufzeit einer frisch alkohol-präzipitierten NS-Fraktion im Viskosimeter wurde das Präparat mit RNase $\left(1 \mathrm{\gamma} / \mathrm{cm}^{3}\right) 30$ Min. bei $37{ }^{\circ} \mathrm{C}$ inkubiert und erneut gemessen. Die Durchlaufzeit hatte sich von ursprünglich 498 Sek. auf 481,7 Sek. verringert, und das Verhältnis $V$ der entsprechenden Viskositäten war $V=0,967$. Anschließend wurde das Präparat mit DNase $\left(1 \gamma / \mathrm{cm}^{3}\right)$ nochmals für 30 Min. bei $37{ }^{\circ} \mathrm{C}$ inkubiert, was einen weiteren Abfall der Durchlaufzeit zur Folge hatte $(V=0,822)$.

Aus diesen Befunden ist zu schließen, daß ein erheblicher Anteil der gesamten inneren Viskosität der NS-Fraktion auf DNS zurückgeht.

Nach erschöpfender RNase- und DNase-Behandlung des Präparates war jedoch die Durchlaufzeit immer noch 80 Sek. länger als diejenige des reinen Lösungsmittels.

Da die innere Viskosität degradierter Nucleinsäure-Moleküle vernachlässigt werden kann, mußte neben RNS und DNS eine weitere Substanz in der NS-Fraktion vorhanden sein (vgl. Chemische Eigenschaften, Abschnitt II).

Der durch enzymatische Spaltung der hochmolekularen NS-Moleküle erzielte prozentuale Abfall der Viskosität gibt ein direktes Maß für den Viskositätsbeitrag beider hochmolekularer Nucleinsäure-Typen.

Aus den chemisch bestimmten tatsächlichen DNSbzw. RNS-Konzentrationen des Präparates ließ sich dann die Viskositätszahl [ $\eta]$ der RNS bzw. DNS nach der angegebenen Formel errechnen.

Diejenige des RNS-Anteils ist mit $[\eta]=95$ von derselben Größe wie diejenige $([\eta]=100)$, die von Gierer z. B. bei RNS aus Mikrosomen von Rattenlebern, ermittelt wurde ${ }^{11}$. Die Viskositätszahl [ $\left.\eta\right]$ für DNS liegt mit 1130 erwartungsgemäß wesentlich höher (s. Tab. 2).

\begin{tabular}{|c|c|}
\hline & Viskositätszahl $[\eta]$ \\
\hline Gesamt-NS $\left(0,86 \mathrm{mg} / \mathrm{cm}^{3}\right)$ & 1120 \\
RNS $\left(0,54 \mathrm{mg} / \mathrm{cm}^{3}\right)$ & 95 \\
DNS $\left(0,32 \mathrm{mg} / \mathrm{cm}^{3}\right)$ & 1130 \\
\hline
\end{tabular}

Tab. 2. Die Viskositäten.

\section{Die Sedimentations-Konstanten}

Bei der Untersuchung von NS-Fraktionen in der analytischen Ultrazentrifuge findet man verschiedene Gradienten.

11 A. Gierer, Z. Naturforschg. 13 b, 788 [1958].
Das Verhältnis von rohen und alkohol-präzipitierten NS-Fraktionen soll gesondert betrachtet werden, da durch die Alkohol-Präzipitation das Verhältnis RNS/DNS und damit die gesamte innere Viskosität der Lösung verändert wird. Bei den rohen NS-Fraktionen waren folgende Gradienten zu beobachten: Ein schneller wandernder Gradient A mit $s_{20} \sim 32 \mathrm{~S}$, ein langsamer wandernder Gradient mit $s_{20} \sim 12 \mathrm{~S} . s_{20}$ des in einem Falle festgestellten Gradienten B liegt zwischen der von $\mathrm{A}$ und $\mathrm{C}$.

Bei den alkohol-präzipitierten NS-Fraktionen wurden folgende Werte ermittelt:

$$
\begin{array}{ll}
\text { Gradient A } & s_{20} \sim 30 \mathrm{~S}, \\
\text { Gradient B } & s_{20} \sim 19 \mathrm{~S}, \\
\text { Gradient C } & s_{20} \sim 10 \mathrm{~S} .
\end{array}
$$

Die gemessenen Sedimentationswerte sind konzentrationsabhängig (s. Tab. 3). Die oben aufgeführten

\begin{tabular}{|l|c|c|c|c|}
\hline Präparat & $\begin{array}{c}\text { Gesamt- } \\
\text { NS } \\
\text { mg } \\
\text { NS/cm }\end{array}$ & $\begin{array}{c}\text { Gradient } \\
\text { A }\end{array}$ & $\begin{array}{c}s_{20} \\
\text { Gradient } \\
\text { B }\end{array}$ & $\begin{array}{c}\text { Gradient } \\
\text { C }\end{array}$ \\
& & & \\
Rohe & 1,86 & 25,5 & 15,3 & 7,8 \\
NS- & 1,94 & 25,0 & & 4,9 \\
Fraktion & 2,84 & 21,8 & & 6,0 \\
alkohol- & 0,95 & 23,7 & & 8,2 \\
präzipi- & 1,54 & 21,4 & & 8,4 \\
tierte & 1,64 & 21,4 & 14,2 & 7,95 \\
NS- & 2,88 & 13,5 & 9,5 & 1,25 \\
Fraktion & 2,90 & 14,5 & 10,9 & 4,85 \\
\hline
\end{tabular}

Tab. 3. Die Sedimentations-Konstanten der Gradienten von rohen und alkohol-präzipitierten NS-Fraktionen.

Werte wurden graphisch auf unendlich kleine NSKonzentrationen extrapoliert.

Die im einzelnen gemessenen Sedimentations-Konstanten in ihrer Abhängigkeit von der Konzentration bei rohen und alkohol-präzipitierten NS-Fraktionen sind in Tab. 3 aufgeführt.

Die für die Gradienten A und B ermittelten Werte stimmen mit denjenigen überein, die Gierer für RNS aus Mikrosomen gemessen hat ${ }^{11}$. Außerdem stimmt die Sedimentations-Konstante des Gradienten A mit der von Gierer für RNS aus TMV gefundenen überein ${ }^{9}$.

Über die chemische Natur der in den drei sichtbaren Gradienten absinkenden Substanzen gab folgendes Experiment Aufschluß:

Eine alkohol-präzipitierte NS-Fraktion mit $2,1 \mathrm{mg}$ $\mathrm{NS} / \mathrm{cm}^{3}$ wurde in der analytischen Ultrazentrifuge 
untersucht. Sie zeigte die drei typischen Gradienten mit den Sedimentations-Konstanten $s_{20} \mathrm{~A}=19,4 \mathrm{~S}$; $s_{20} \mathrm{~B}=12,6 \mathrm{~S} ; s_{20} \mathrm{C}=6,4 \mathrm{~S}$ (s. Abb. 2 ).

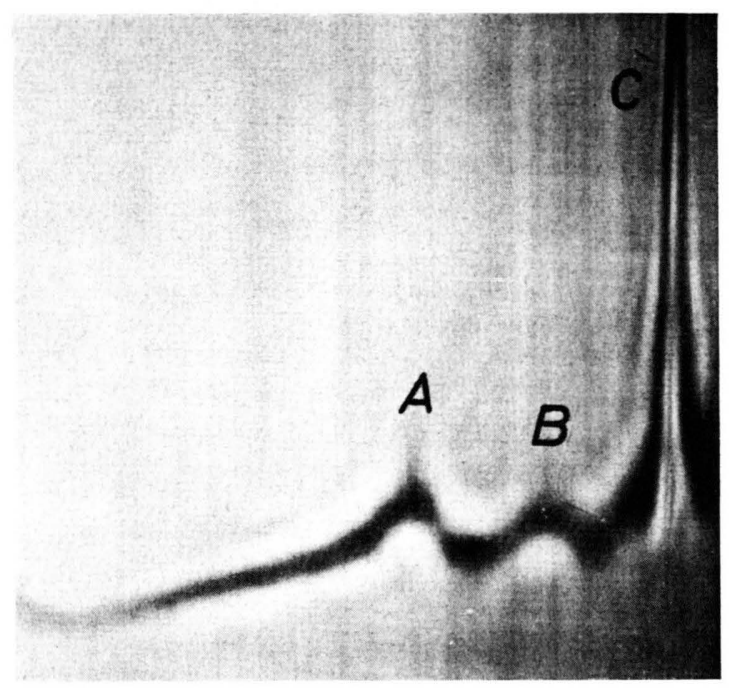

Abb. 2.

Abb. 2-4: Sedimentationsdiagramme einer alkohol-präzipitierten NS-Fraktion. Abb. 2: Nicht mit Enzym behandelt, Abb. 3: nach Behandlung mit RNase, Abb. 4: nach Behandlung mit DNase.

Eine Probe derselben NS-Fraktion wurde mit $1 \gamma$ RNase $/ \mathrm{cm}^{3}$ für 10 Min. bei Raumtemperatur behandelt. Bei der anschließenden Zentrifugation zeigte sich, daß die Gradienten A und B vollständig verschwunden waren. Der unverändert große Gradient $\mathrm{C}$ hatte eine Sedimentations-Konstante $s_{20}=7,9 \mathrm{~S}$ (s. Abb. 3).

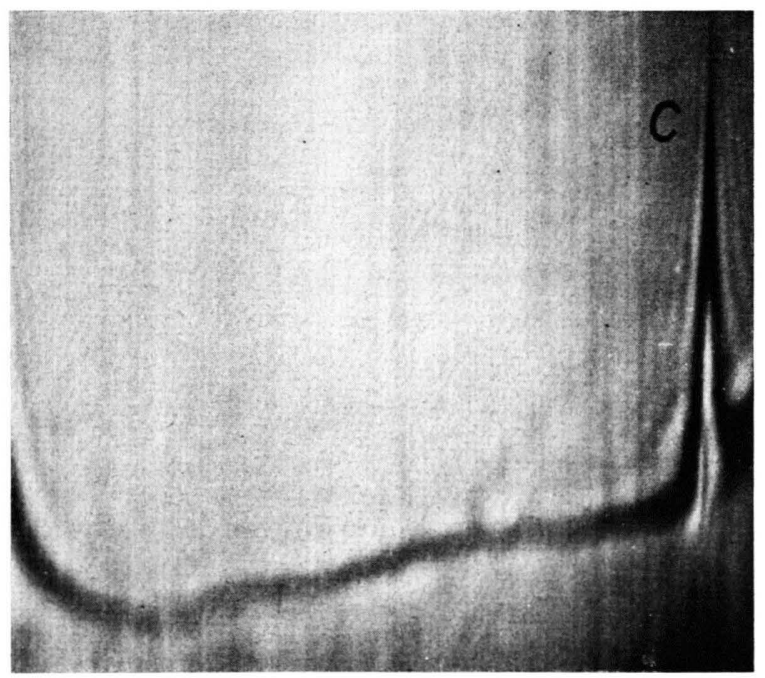

Abb. 3.
Wurde das Material dagegen mit $1 \gamma \mathrm{DNase} / \mathrm{cm}^{3}$ für 60 Min. bei Raumtemperatur vorbehandelt, so blieben die Gradienten A und B optisch unverändert und sedimentierten mit $s_{20} \mathrm{~A}=21,5 \mathrm{~S}$ bzw. $s_{20} \mathrm{~B}$ $=11,4 \mathrm{~S}$. Der Gradient $\mathrm{C}$ aber wurde dadurch wesentlich abgeflacht und verbreitert, wenn er auch nicht ganz verschwunden war (s. Abb. 4).

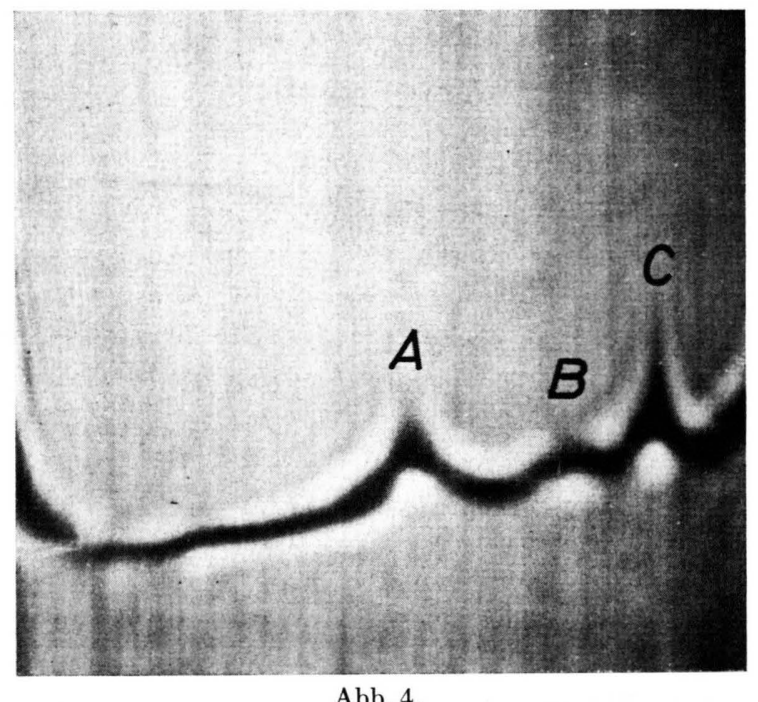

Abb. 4 .

Die Gradienten A und B gehen also auf RNS, der Gradient C auf DNS zurück. Letzterer unterliegt auf Grund der hohen Viskosität des Materials einem starken Selbstschärfungs-Effekt. Berechnet man aus dem Flächeninhalt der drei Gradienten die relative Konzentration der betreffenden Substanzen, so ergibt sich für A ca. $70 \%$, für $\mathrm{B}$ ca. $5 \%$ und für $\mathrm{C}$ ca. 25 Prozent.

In einem weiteren Versuch wurden eine alkoholpräzipitierte NS-Fraktion mit $1,9 \mathrm{mg} \mathrm{NS} / \mathrm{cm}^{3}$ und eine RNS aus TMV mit $1,3 \mathrm{mg} \mathrm{RNS} / \mathrm{cm}^{3}$ zu gleichen Teilen gemischt.

Bei der Untersuchung dieses Mischpräparates in der analytischen Ultrazentrifuge war neben den Gradienten $\mathrm{A}, \mathrm{B}$ und $\mathrm{C}$ auch bei längerem Zentrifugieren kein weiterer Gradient festzustellen. Wohl aber war der Flächeninhalt des Gradienten A vergrößert. Auch diese Feststellung spricht dafür, daß die RNS A der NS-Fraktionen und die RNS aus TMV die gleichen Sedimentations-Eigenschaften haben.

Nachdem das Verhalten der NS-Fraktionen in der analytischen Ultrazentrifuge bekannt war, blieb die Frage zu klären, ob man das infektiöse Prinzip einem der drei beobachteten Gradienten zuordnen kann. 


\begin{tabular}{|c|c|c|c|c|c|c|c|}
\hline \multirow[b]{3}{*}{ Präparat } & \multicolumn{5}{|c|}{$\begin{array}{c}\text { Abfall } \\
{[\%]}\end{array}$} & & \\
\hline & \multicolumn{2}{|c|}{ analytische $\mathrm{UZ}^{*}$} & \multicolumn{2}{|c|}{$\begin{array}{c}\text { Soll } \\
\text { Infektiositäts- } \\
\text { Verlust }\end{array}$} & \multirow[t]{2}{*}{$\begin{array}{c}\text { Ist } \\
\text { Infektiositäts- } \\
\text { Verlust }\end{array}$} & \multicolumn{2}{|c|}{$\begin{array}{c}\text { Abweichungen } \\
{[\%]}\end{array}$} \\
\hline & $s_{20} \mathrm{~A}$ & $s_{20} \mathrm{~B}$ & A & B & & zu A & zu B \\
\hline $\begin{array}{l}4 \mathrm{~cm}^{3} 40705 \mathrm{U} / \mathrm{Min} . \\
55 \mathrm{Min} .+5^{\circ} \mathrm{C} \\
2,9 \mathrm{mg} \mathrm{NS} / \mathrm{cm}^{3}\end{array}$ & 13,5 & 9,5 & 38 & 19 & 30 & -8 & +11 \\
\hline $\begin{array}{l}4 \mathrm{~cm}^{3} 39500 \mathrm{U} / \mathrm{Min} . \\
60 \mathrm{Min} .+5^{\circ} \mathrm{C} \\
2,1 \mathrm{mg} \mathrm{NS} / \mathrm{cm}^{3}\end{array}$ & 19,4 & 12,6 & 63 & 35 & 92 & +29 & +57 \\
\hline $\begin{array}{l}4 \mathrm{~cm}^{3} 40800 \mathrm{U} / \mathrm{Min} . \\
60 \mathrm{Min} .+5^{\circ} \mathrm{C} \\
086 \mathrm{mg} \mathrm{NS} / \mathrm{cm}^{3}\end{array}$ & 26 & 16,4 & 83 & 48 & 96 & +13 & +48 \\
\hline $\begin{array}{l}1,5 \mathrm{~cm}^{3} 39000 \mathrm{U} / \mathrm{Min} . \\
130 \mathrm{Min} .18^{\circ} \mathrm{C} \\
2,1 \mathrm{mg} \mathrm{NS} / \mathrm{cm}^{3} \\
\text { Schwingrotor }\end{array}$ & 19,4 & 12,6 & 100 & 86 & 96 & -4 & +10 \\
\hline Durchschnitt & & & & & & $+7,5$ & $+31,5$ \\
\hline
\end{tabular}

* UZ $=$ Ultrazentrifugation.

Tab. 4. Verhalten der Gradienten A und B und der Infektiosität von NS-Fraktionen beim Zentrifugieren.

Es wurde deshalb versucht, aus dem Abfall der Infektiosität nach hochtouriger Zentrifugation des Materials die Sedimentations-Konstante des infektiösen Prinzips der NS-Fraktion zu ermitteln.

Hierzu wurden verschiedene Experimente durchgeführt: In einer Versuchsserie wurden zunächst die Sedimentations-Konstanten für die drei verschiedenen Gradienten einer bestimmten NS-Fraktion optisch gemessen. Gleichzeitig damit wurden jeweils $4 \mathrm{~cm}^{3}$ desselben Materials im Rotor 40 der Spinco-Zentrifuge bei $+5{ }^{\circ} \mathrm{C} 55$ oder 60 Min. mit $40000 \mathrm{U} /$ Min. zentrifugiert und der Abfall der Infektiosität der Überstände gegenüber derjenigen des Ausgangsmaterials im Eitest bestimmt (Verdünnungen nach Potenzen von 5, 10 Eier/Verdünnung). Aus den bekannten Sedimentations-Konstanten der drei Gradienten und den bei der präparativen Zentrifugation angewendeten Zentrifugations-Daten ließ sich mit ausreichender Genauigkeit errechnen, wieviel Prozent der einzelnen Substanzen aus dem UUberstand sedimentiert sein mußten ${ }^{12}$. Der Vergleich dieser Werte mit den durch Infektionstest ermittelten Werten ergibt, wie Tab. 4 zeigt, eine relativ gute Übereinstimmung der biologisch ermittelten SedimentationsEigenschaften mit denjenigen des Gradienten A.

In einem ähnlichen Versuch wurden $1,5 \mathrm{~cm}^{3}$ einer NS-Fraktion im Schwingrotor der Spinco-Zen-

12 P. Giebler, Z. Naturforschg. 13 b, 238 [1958]. trifuge 130 Min. mit $39000 \mathrm{U} /$ Min. zentrifugiert. Die Temperatur am Ende der Zentrifugation betrug $+18{ }^{\circ} \mathrm{C}$. Die oberen $2 / 3$ wurden dann vorsichtig abgesaugt und im Infektionstest geprüft.

Das Ergebnis ist ebenfalls in Tab. 4 gezeigt. Es ergibt sich auch hier eine gute Übereinstimmung der gemessenen mit den für Gradient A errechneten Werten.

Schließlich wurde in zwei Versuchen eine NS-Fraktion in der analytischen Ultrazentrifuge solange zentrifugiert, bis der Gradient A gerade vollständig sedimentiert war. Dann wurden $80 \%$ des Gesamtüberstandes vorsichtig abgeschichtet und, wegen des geringen Volumens, nur qualitativ auf Infektiosität geprüft. Dasselbe wurde mit dem Überstand nach 70-proz. Sedimenation des Gradienten A durchgeführt. Die dabei ermittelten Werte sind folgende:

\begin{tabular}{|c|c|c|c|}
\hline & \multicolumn{2}{|c|}{$\begin{array}{l}\text { sedimentiert } \\
{[\%]}\end{array}$} & \multirow[t]{2}{*}{$\begin{array}{l}\text { Infektiosität der oberen } \\
80 \% \text { des Úberstandes }\end{array}$} \\
\hline & A & B & \\
\hline 1. & 100 & 70 & negativ \\
\hline 2. & 70 & 40 & positiv \\
\hline
\end{tabular}

Aus allen diesen Versuchen zur biologischen Bestimmung der Sedimentations-Konstanten des infektiösen Prinzips der NS-Fraktionen ergibt sich ein Wert, der innerhalb der für den RNS-Gradienten A 
ermittelten Größenordnung liegt. Daraus ergibt sich dann weiter unter Berücksichtigung der Viskositätszahl des RNS-Anteils der NS-Fraktionen ein wahrscheinliches Mol.-Gew. für die infektiöse Komponente von ca. $2 \cdot 10^{6}$, innerhalb der Grenzen ca. $1 \cdot 10^{6}$ bis $3 \cdot 10^{6}$.

\section{Chemische Eigenschaften der NS-Fraktionen \\ 1. Das Verhalten gegenüber RNase und DNase}

Nachdem im vorhergehenden Kapitel die physikalischen Daten ergaben, daß sowohl RNS wie DNS in den NS-Fraktionen enthalten waren, und daß das infektiöse Prinzip sehr ähnliche SedimentationsEigenschaften besaß, wie sie von Gierer für die RNS aus TMV beschrieben wurden, war es notwendig, die Empfindlichkeit des infektiösen Prinzips von NS-Fraktionen gegenüber RNase und DNase eingehend zu prüfen. Aus den Ergebnissen mußte sich dann im Zusammenhang mit den physikalischen Eigenschaften des infektiösen Agens der NS-Fraktionen ergeben, ob es sich hierbei um RNS- oder DNSMoleküle handelt.

Einzelne Verdünnungen einer NS-Fraktion wurden mit jeweils 3 verschiedenen Konzentrationen von DNase und einer Konzentration von RNase für 15 Min. bei $+10^{\circ} \mathrm{C}$ inkubiert und anschließend im Infektionstest geprüft. Dieser wurde mit je 10 Eiern/NS-Fraktions-Verdünnung und pro EnzymKonzentration durchgeführt. Beide Verdünnungsreihen waren nach Potenzen von 10 angesetzt.

\begin{tabular}{|l|c|}
\hline \multicolumn{1}{|c|}{ Behandlung } & $\log L D_{50} / \mathrm{cm}^{3}$ \\
\hline Kontrolle & 2,86 \\
RNase $0,01 \gamma / \mathrm{cm}^{3}$ & 0 \\
DNase $1,0 \gamma / \mathrm{cm}^{3}$ & 3,45 \\
DNase $0,1 \gamma / \mathrm{cm}^{3}$ & 3,13 \\
DNase $0,01 \gamma / \mathrm{cm}^{3}$ & 3,39 \\
\hline
\end{tabular}

Tab. 5. Der Einfluß von RNase und DNase auf die Infektiosität einer NS-Fraktion.

Das Ergebnis ist in Tab. 5 gezeigt. Die Behandlung mit RNase hatte den völligen Verlust der Infektiosität zur Folge, während sämtliche geprüften DNase-Konzentrationen die Infektiosität gegenüber der Kontrolle sogar noch etwas zu steigern schienen. In einem weiteren Versuch wurde festgestellt, daß bei gleicher Inkubations-Temperatur und -Dauer
$0,001 \gamma \mathrm{RNase} / \mathrm{cm}^{3}$ die Infektiosität einer NS-Fraktion zwar nicht mehr vollständig, aber immer noch um $60 \%$ zu zerstören vermögen.

RNase besitzt also eine um wenigstens 1000-mal größere Wirkung auf die Infektiosität als DNase, woraus sich ergibt, daß das infektiöse Material in den NS-Fraktionen aus RNS besteht, die zumindest in einer außerordentlich RNase-empfindlichen Anordnung vorliegen muß.

\section{Die chemische Zusammensetzung der NS-Fraktionen}

\section{a) Die Reaktion auf Desoxyribose}

Auf Grund der Viskositäts- und SedimentationsMessungen war schon das Vorhandensein von DNS in den NS-Fraktionen ermittelt worden. Durch die Ausmessung der relativen Anteile der drei Gradienten, die in der analytischen Ultrazentrifuge beobachtet werden können, war für die Bande C, die auf DNS zurückgeht, eine Konzentration von etwa 25\% der Gesamt-NS vermutet worden.

Genauere Daten über den DNS-Gehalt von NSFraktionen wurden durch die Reaktion mit D is ches Reagens gewonnen, bei der die Desoxyribose bestimmt wird (s. Material und Methoden). Sämtliche damit geprüften NS-Fraktionen hatten einen DNSGehalt, der bei rohen Präparaten um 25\%, bei alkohol-präzipitierten NS-Fraktionen durchschnittlich bei $37 \%$ lag. Diese Zahlen beziehen sich auf den Gewichtsanteil der DNS an der gesamten NucleinsäureMenge, die auf Grund der Extinktion der NS-Fraktionen bei $258 \mathrm{~m} \mu$ errechnet wurde.

Die Auswirkung der Alkohol-Präzipitation auf die DNS-Konzentration der NS-Fraktionen ist in Tab. 4 gezeigt. Durch die Alkohol-Präzipitation tritt praktisch kein Verlust an DNS auf, dagegen nimmt die restliche Nucleinsäure, die als RNS angenommen werden darf, um durchschnittlich etwa $40-50 \%$ ab.

\section{b) Die Eiweiß-Reaktion}

Die Messung der UV-Absorption von NS-Fraktionen, die Bestimmung des Extinktions-Verhältnisses E 258/E $280 \mathrm{~m} \mu$ sowie der Vergleich zwischen berechnetem und gefundenem Stickstoff-Gehalt hatten angezeigt, daß in den rohen NS-Fraktionen ein stickstoff-haltiges Material vorkommt, das nicht NucleotidCharakter besitzt. Durch die Fällung der NS-Fraktionen mit Alkohol konnte dieses Material weitgehend entfernt werden.

Die modifizierte Biuret-Reaktion (s. Material und 
Methoden) gibt nach Schuster bei einer Mindestmenge von $50 \gamma$ Protein $/ \mathrm{cm}^{3}$ noch einen positiven Ausfall ${ }^{13}$.

Sämtliche geprüften rohen NS-Fraktionen zeigten einen positiven Ausfall der Reaktion, während bei sämtlichen geprüften alkohol-präzipitierten NS-Fraktion die Reaktion negativ verlief. Legt man die gesamte NS-Menge einer Fraktion als Bezugswert zugrunde, so ergibt sich in jedem Fall, daß die alkoholpräzipitierten Fraktionen weniger als $2 \%$ Protein besitzen. Die höchste hierbei untersuchte NS-Konzentration einer alkohol-präzipitierten Fraktion war $2,98 \mathrm{mg} \mathrm{NS} / \mathrm{cm}^{3}$, was also bei negativem Ausfall der Biuret-Reaktion einer maximalen Protein-Verunreinigung von weniger als $1,7 \%$ entspricht.

Durch diese Daten wird die Vermutung gestützt, daß es sich bei den stickstoff-haltigen Verunreinigungen roher NS-Fraktionen um Aminosäuren oder kleine Peptide handelt, die durch Alkohol nicht präzipitiert und deshalb von den fällbaren NucleinsäureMolekülen getrennt werden können.

\section{c) Die Zucker-Reaktion}

Bei der Viskositäts-Messung hatte es sich gezeigt, daß selbst nach mehrstdg. Inkubation einer NS-Fraktion mit DNase und RNase eine Rest-Viskosität zurückblieb, die auf andere visköse Substanzen als NS zurückgehen mußte. Protein war weitgehend auszuschließen, da es sich um eine durch Alkohol-Präzipitation gereinigte Fraktion gehandelt hatte.

Andererseits gehen aber bei der Behandlung mit Phenol auch hochpolymere Zucker z. T. in die wäßrige Phase über ${ }^{14}$. Es war also durchaus wahrscheinlich, daß neben RNS und DNS auch noch hochmolekulare Polysaccharide vorhanden waren.

\begin{tabular}{|c|c|c|c|c|c|c|}
\hline & & $\begin{array}{l}\text { Gesamt- } \\
\mathrm{NS} \\
{\left[\gamma / \mathrm{cm}^{3}\right]}\end{array}$ & $\begin{array}{c}\text { DNS } \\
{\left[\gamma / \mathrm{cm}^{3}\right]}\end{array}$ & \begin{tabular}{|c|}
$\begin{array}{c}\text { DNS- } \\
\text { Gehalt }\end{array}$ \\
{$[\%]$}
\end{tabular} & $\begin{array}{c}\mathrm{RNS} \\
{\left[\gamma / \mathrm{cm}^{3}\right]}\end{array}$ & $\begin{array}{l}\text { Ver- } \\
\text { lust } \\
\text { RNS } \\
{[\%]}\end{array}$ \\
\hline Vor & $\begin{array}{l}\text { Alkohol- } \\
\text { Präzipi- }\end{array}$ & 2588 & 615 & 24 & 1973 & \\
\hline Nach & tation & 1822 & 606 & 33 & 1216 & 38 \\
\hline Vor & $\begin{array}{l}\text { Alkohol- } \\
\text { Präzipi- }\end{array}$ & 4180 & 1150 & 27,5 & 3030 & \\
\hline Nach & & 2510 & 1000 & 40 & 1510 & 50 \\
\hline
\end{tabular}

Tab. 6. Gesamt-NS, DNS- und RNS-Anteil von NS-Fraktionen und der Einfluß der Alkohol-Präzipitation.

${ }^{13}$ H. Schuster, W. Zillig u. G. Schrama, Z. Naturforschg. 11 b, 339 [1956].
$\mathrm{Zu}$ ihrem Naweis beschränkten wir uns auf eine grob quantitative Bestimmung des Gesamt-ZuckerGehaltes einer NS-Fraktion nach der Methode von Tillmanns und Philippi (s. Material und Methoden).

Bei einer alkohol-präzipitierten NS-Fraktion mit einer gesamten NS-Konzentration von $2,5 \mathrm{mg} / \mathrm{cm}^{3}$ wurden $1,9 \mathrm{mg}$ Glucose-Äquivalente $/ \mathrm{cm}^{3}$ gefunden. Durch die Ribose und Desoxyribose der Nucleinsäuren waren aber nur $60 \%$ davon, nämlich $1,16 \mathrm{mg}$ Glucose-Äquivalente $/ \mathrm{cm}^{3}$ gedeckt, wenn für beide Nucleinsäure-Typen ein Gewichtsanteil des Zuckers von $46 \%$ angenommen wird. Der gesamte Zuckergehalt der NS-Fraktion überstieg also den auf $\mathrm{Nu}$ cleinsäure-Zucker zurückgehenden Wert um 40 Prozent. Diese Polysaccharide müssen hochmolekular sein, da sie erstens eine relativ hohe Viskosität besitzen und zweitens durch Alkohol-Präzipitation nicht entfernt werden.

\section{Diskussion}

In einer früheren Mitteilung hatten wir über ein Verfahren berichtet, aus Gehirnen von Mäusen, die an einer Infektion mit EEE-Virus zugrunde gegangen waren, mittels Phenol in der Kälte eine nucleinsäure-haltige Fraktion zu gewinnen, die infektiös ist, aber sehr wahrscheinlich keine intakten Virusteilchen mehr enthält ${ }^{4}$.

Es wurde nun festgestellt, daß solche nucleinsäurehaltigen Fraktionen sich auch aus virus-infizierten Hühnerembryonen extrahieren lassen. Die leichte Gewinnung beliebiger Mengen dieses Ausgangsmaterials ermöglichte detailliertere Untersuchungen über die physikalischen und chemischen Eigenschaften solcher NS-Fraktionen.

In der analytischen Ultrazentrifuge waren drei Gradienten zu beobachten, die im Text mit A, B und $\mathrm{C}$ bezeichnet sind. A und B gehen auf RNS, C auf DNS zurück. Bei Extrapolation der SedimentationsKonstanten $c \rightarrow 0$ ergaben sich folgende Werte für die drei Gradienten:

$$
\mathrm{A} \sim 30-32 \mathrm{~S} ; \mathrm{B} \sim 19 \mathrm{~S} ; \mathrm{C} \sim 12 \mathrm{~S} .
$$

Die Ribonucleinsäuren A und B stimmen nach ihrem Verhalten im Schwerefeld mit denjenigen überein, die GIERER ${ }^{11}$ neuerdings aus Mikrosomen von Rattenlebern und normalen Tabakblättern isoliert hat. Auch die Viskositätszahl seiner Präparate ist mit

14 O. Westphal, O. Lüderitz u. F. Bister, Z. Naturforschg. 7 b. 148 [1952]. 
$[\eta]=100$ sehr ähnlich mit dem Wert von $[\eta]=95$, der für die Gesamt-RNS unserer NS-Fraktionen ermittelt wurde. Außer den beiden Ribonucleinsäuren $\mathrm{A}$ und $\mathrm{B}$ fanden sich noch kleinmolekulare Ribonucleotide in den Fraktionen. Diese repräsentieren etwa $40-50 \%$ der gesamten RNS.

Im Gegensatz zum RNS-Anteil scheint die DNS in den Fraktionen von einheitlich hohem Mol.-Gew. zu sein.

Neben RNS- und DNS-Fraktionen konnten noch folgende Komponenten nachgewiesen werden:

1. Ein stickstoff-haltiges, biuretpositives, kleinmolekulares Material, das im UV-Licht von $280 \mathrm{~m} \mu$ Wellenlänge absorbiert und als aus Aminosäuren oder kleinen Peptiden bestehend angesehen wird. Es enthält etwa $40-50 \%$ des Gesamt-Stickstoffes einer NS-Fraktion.

2. Eine hochmolekulare, visköse Zuckerkomponente, die $40 \%$ der gesamten Glucose-Äquivalente einer NS-Fraktion darstellt.

Versetzt man NS-Fraktionen mit 2 Volumenteilen Äthanol, so werden die Ribonucleinsäuren A und B, die gesamte DNS sowie die Zuckerkomponente präzipitiert, während die kleinmolekularen Ribonucleotide und das biuretpositive Material im Überstand bleiben. Die Präzipitate lösten sich leicht in 0,02-m. Phosphatpuffer und ergaben die sogenannten ,alkohol-präzipitierten NS-Fraktionen“, in denen keine Verunreinigungen mit Protein nachgewiesen werden konnten. Ihr chemisch ermittelter Stickstoff stimmte praktisch vollkommen mit dem überein, der sich aus der Extinktion im UV-Licht bei einer Wellenlänge von $258 \mathrm{~m} \mu$ errechnen läßt.

Das Hauptinteresse der Untersuchungen konzentrierte sich auf den RNS-Anteil A der NS-Fraktio-

15 E. Chargaff u. J. N. Davidson, in: The Nucleic Acids, Volume II, New York 1955.

16 A. Gierer, Z. Naturforschg. 13 b, 485 [1958]. nen, da sein physikalisches Verhalten außerordentlich ähnlich mit demjenigen infektiöser RNS aus TMV ist $^{9,16}$. Tatsächlich zeigte die Mischung einer NSFraktion und einer infektiösen RNS aus TMV zu gleichen Teilen keinen zusätzlichen Gradienten in der analytischen Ultrazentrifuge und der etwas ausgeprägtere Gradient A blieb einheitlich. Durch Verfolgen der Sedimentation des infektiösen Prinzips konnte nun wahrscheinlich gemacht werden, daß dieses im Gradienten A enthalten ist. Da das infektiöse Prinzip außerordentlich empfindlich gegenüber RNase ist, darf es wohl als RNS der entsprechenden Größe bezeichnet werden. Diese ergab sich aus der Sedimentations-Konstante für A $=30-32 \mathrm{~S}$ und der Viskositätszahl für die Gesamt-RNS der NS-Fraktionen $[\eta]=95$. Danach besitzt die RNS A und damit wahrscheinlich auch die infektiöse RNS ein Mol.Gew. von etwa $2 \cdot 10^{6}$, die RNS B ein solches von etwa $0,6 \cdot 10^{6}$.

Die Übereinstimmung mit den Mol.-Gew., die von Gierer für die infektiöse RNS aus Tabakmosaik-Virus (TMV) ${ }^{9,16}$ und die RNS aus Mikrosomen und normalen Tabakblättern ${ }^{11}$ nach derselben Methode ermittelt wurden, ist auffällig. Es ergibt sich aus diesen Versuchen, daß die infektiöse RNS des tierpathogenen EEE-Virus ein sehr ähnliches Mol.-Gew. zu besitzen scheint, wie die infektiöse RNS aus dem pflanzenpathogenen TMV.

Herrn Professor Dr. W. SchäFER danke ich für sein stetes Interesse und die von ihm erfahrene Förderung der Arbeit, Herrn Dr. A. Gierer für seine wertvollen Hinweise, Diskussionen und Ratschläge, Herrn P. GiebLER für die Durchführung und Berechnung der Experimente zur Bestimmung der Sedimentations-Konstanten. Meiner langjährigen Assistentin Frau I. Mussgay bin ich für ihre unermüdliche Hilfe bei der Durchführung der Arbeit zu besonderem Dank verpflichtet. Die Arbeit wurde mit Mitteln der Deuts chen Fors chungs. g e m e in s ch a f $t$ durchgeführt. 